Published in final edited form as:

Otol Neurotol. 2018 June ; 39(5): e387-e391. doi:10.1097/MAO.0000000000001801.

\title{
Temporal Bone Histopathology in Cockayne Syndrome
}

\author{
Ophir Handzel, MD ${ }^{1}$ and Joseph B Nadol Jr., MD ${ }^{2}$ \\ ${ }^{1}$ Director Cochlear Implant Center, Department of Otolaryngology/Head, Neck \& Maxillofacial \\ Surgery, Tel-Aviv Sourasky Medical Center, Sackler Faculty of Medicine, Tel-Aviv, Israel \\ 2Otopathology Laboratory, Department of Otolaryngology, Massachusetts Eye and Ear Infirmary, \\ Harvard Medical School, Boston, Massachusetts
}

\section{Introduction}

Cockayne Syndrome (CS) is a devastating neuro-developmental autosomal recessive disorder, which includes premature aging, dwarfism, central nervous system degeneration, and non-cancerous hyper-sensitivity to the sun. Hearing loss (HL) is characteristic of the syndrome. It is sensorineural, bilateral, and progressive commonly resulting in profound HL. Nance and Berry classified CS patients into 3 clinical subtypes based on the age of presentation and the severity of disease (1). Type 1 is the classical form presenting in the first two years of life, type 2 is congenital and more severe, and type 3 has a later onset and a milder course.

There are two type of genetic abnormalities resulting in CS, divided into complementation group A (20\% of cases) and B (80\% of cases)(2). Each group represents a different mutation in excision repair cross complementation (ERCC) genes. ERCC6 encodes the CSB protein. CSB is a component of the transcription-coupled nucleotide excision repair pathway (3) and RNA polymerase $1(4,5)$. In addition, both CSA and CSB are involved in the repair of DNA defects caused by oxygenation. CSB related genes are found in both the nucleus and the mitochondria.

CS is rare $(2: 1,000,000)$ and temporal bones specimens accompanied with clinical information and genetic testing gathered during life are exceedingly rare. Here we present the temporal bone findings in a patient with CSB clinical type 1 .

\section{Material Methods}

\section{Temporal Bone Acquisition}

Temporal bone specimens were obtained in compliance with Human Subject Assurance No. FWA00006221 (exemption 4) from the Massachusetts Eye and Ear Infirmary.

\section{Temporal Bone Preparation and Light Microscopy}

Only the right temporal bone was available for study. The time elapsed between death of the donor to tissue fixation was 4 hours. The bone was fixed in $10 \%$ formalin, decalcified with ethylene diamine tetra-acetic acid (EDTA), dehydrated in graded alcohols and embedded in celloidin. Serial sections $20 \mu \mathrm{m}$ thick were cut in the axial (horizontal) plane. Every tenth 
section was stained with hematoxylin and eosin (H\&E) and mounted on glass slides. For analysis and quantification of cellular elements two dimensional reconstruction of the cochlea was done. Neurons were counted by identification of their nucleoli under 400x magnification. A correction factor of 0.9 was applied to the counted number of neurons to account for the possibility of double counting in two consecutive sections (6).

\section{Results \\ Clinical History}

This 29 year old man had a history of developmental delay, seizure disorder, renal insufficiency and secondary hypertension. He was tracheotomy and ventilator dependent in late stages of his life and died of respiratory failure. The diagnosis of Cockayne syndrome was made based on clinical features and genetic testing that revealed a homozygous missense variant c. 1820A>G (p. K607R) in the ERCC6 gene. This variant is compatible with CS complementation group B. The clinical presentation is compatible with subtype 1 .

Five years prior to death the patient reported experiencing non-pulsatile tinnitus. An audiogram three and a half years before his death documented a moderate symmetrical sensorineural hearing loss at $2-8 \mathrm{kHz}$ (Figure 1). Speech reception threshold was $20 \mathrm{~dB}$ and the word recognition score (CID W22) was $100 \%$ on the right, and $25 \mathrm{~dB}$ and $92 \%$ respectively in the left ear. There was no history of vestibular complaints.

\section{Histopathological Findings}

Organ of Corti and cochlear neurons-There was patchy loss of inner and outer hair cells particularly at the base of the cochlea (Fig 1). Significant atrophy of cellular elements of the spiral ligament, stria vascularis and spiral prominence was present (Figs 1-4). There was loss of Claudius cells, outer sulcus cells, and mesenchymal cells on the scala tympani side of the basilar membrane (Fig 4). There was loss of some interdental cells in the limbus (Figure 4). There was a moderate loss of neurons of the spiral ganglion (Figs 1-3). The total number of spiral ganglion neurons was 13455 , representing $43 \%$ of the normal age-matched complement.

Vestibular System-The neuroepithelium of the five vestibular sense organs was intact other than some hair cell loss in the saccule. The perilymphatic and endolymphatic spaces of the vestibular membranous labyrinth were partially occupied by fibrillar material and new bone formation was found in the perilymphatic space most pronounced in the lateral semicircular canal (Fig 5).

The number of Scarpa's ganglion neurons was moderately reduced at 11519 neurons representing 59\% of the age matched norm. The superior division had 6213 neurons, and the inferior division 5306, representing 53\% and $67 \%$ of the age-matched normal respectively.

\section{Discussion}

CS is a genetically determined progressive neurodegenerative syndrome leading to accelerated aging and hearing loss. The diagnosis is made based on clinical presentation and 
verified by genetic testing. Growth failure, microcephaly, and developmental delay have been considered required for the diagnosis. Recently, Wilson et al. has suggested that the diagnosis should be suspected in any child with postnatal growth failure, microcephaly and two of a number of minor criteria that include hearing loss (7). CS is a rare disease, with a prevalence of 2.5 per million (8). However, in a few isolated populations it is more common (i.e. in one particular village in Israel 1:15 are carriers of an ERCC6 mutation) (9).

Hearing loss is a common feature of the syndrome. It is almost always bilateral, and presents neonatally in $21 \%$ of patients and in $84 \%$ by the age of 10 years (7). Sensorineural hearing loss is considered a minor criterion for the diagnosis of CS. Hearing loss may be conductive or mixed conductive and sensorineural hearing loss in up to $44 \%$ of patients (7). The cause of a conductive component is unknown. Delayed and mild forms of hearing of hearing loss have been reported (10). Histopathology analysis has not revealed a potential cause of a conductive component.

The specimen described herein demonstrated mild degeneration of the organ of Corti and is only the second case studied that had an accompanying behavioral audiogram. The most severe degeneration was found in the stria vascularis, spiral ligament and spiral prominence with only moderate neural loss in the spiral and Scarpa's ganglions.

Shemen reported three members of a single family with CS (11). One of patients had a temporal bone available for study but no accompanying audiogram. That bone showed loss of hair cells and spiral ganglion neurons most pronounced in the basal turn. Gandolfi et al. reported similar findings in a 17 year old patient (12). Rapin and her colleagues presented the histology of the CNS, eye and inner ear of a patient with CS type A who died at the age of 31 years (13). There was diffuse degeneration of the pars inferior including hair cells, spiral ganglion cells, stria vascularis and other supporting structures, and an audiogram demonstrated anacusis in both ears. Since the previously published cases had end stage disease in terms of inner ear structure and function it is hard to conclude whether neural loss in these was primary or secondary to the loss of hair cells. In contrast, the case reported herein demonstrated only moderate hearing loss and corresponding histopathology. These findings suggest that CSB neuronal loss is not a primary event but rather secondary to the loss of other structures.

In contrast to hearing loss vestibular symptoms have not been routinely reported. Some patients suffered from ataxia that presumably originated from CNS abnormalities. In this case there was no mention in the medical records of vestibular symptoms. There was mild loss of sacular neuroepithelium, fibrosis of the endolymphatic and perilymphatic spaces and ossification of the perilymphatic space. The fibrosis and new bone formation were seen in one previously published case (13). To the best of our knowledge no case has been reported with in vivo functional vestibular testing. In one case there was collapse of the endolymphatic duct of the pars inferior but not of the pars superior (13). There was severe loss of hair cells in the pars superior (utricle and semicircular canals) that affected all three cristae, with a few hair cells still present in the utricle. Neurons in Scarpa's (vestibular) ganglion were markedly depleted, with loss of peripheral dendritic processes. Dark cells of 
the vestibular labyrinth were still present. One of the patients reported by Shemen et al. had nystagmus but no additional details are provided (11).

The pathogenesis of the phenotype seen in CS involves a number of pathways. CSB is defined by mutations in the ERCC6 gene that codes for a protein that functions in the transcription-coupled repair of damaged DNA, RNA synthesis, and mitochondrial metabolism. CS has traditionally been thought of as a disorder caused by defective transcription and transcription-coupled nucleotide excision repair (TC-NER) (8). Deficient transcription-coupled nucleotide excision repair is responsible for at least the sun sensitivity (14). However, TC-NER can't explain all the features seen in CS. Abnormal RNA polymerase I and II are likely to play a role in other symptoms of the disease including hearing loss (14). Recently, it has been shown that $C S A$ and $C S B$ deficiency related impaired DNA repair results in hair cell (rather than neuronal) loss [15]. A possible mechanism is abnormal sensitivity to insults rather than damage directly caused by the mutated gene products [15]. Indeed $C S A$ and $C S B$ mutated mice manifest excessive sensitivity to cisplatin [16]. Stains for anti-NF, anti-MPZ, and Gomori trichrome in the presented specimen were inconclusive for myelinopathy. CS and xeroderma pigmentosum (XP) probably share some of these mechanisms. In both conditions patients suffer from defective DNA repair of damage induced by sun exposure and probably reactive oxygen species. Histopathology of the temporal bones of patients with XPD and XPA has been described (16). Main findings in XP were atrophy of the organ of Corti, stria vascularis, and cochlear neurons. The latter could be primary or secondary.

Many of the clinical features seen in CS are also observed in mitochondrial disorders (8). CSA and CSB are expressed in the mitochondria, and abnormalities in this organelle are likely responsible for the loss of subcutaneous tissue seen in CS (16). Neural degeneration can be attributed to reduced synaptic density caused by dysregulation of the growth hormone/Insulin-like growth factor 1 pathway and pathways related to synapse formation, maintenance and neural differentiation (5).

The finding in the current case that most pronounced cochlear damage was in the stria vascularis and spiral ligament and not to the spiral ganglion leads to a conclusion that cochlear implantation should be beneficial to patients with CS who met the criteria for implantation, even in more advanced stages of the disease and more severe HL. Indeed several individuals have been reported to have received benefit from cochlear implants (7).

Impaired mitochondrial function in CSB patient-derived cells has been successfully repaired (4). Imperfect neuronal differentiation and neuritogenesis in cell culture was successfully bypassed by treatment with amitriptyline or 7, 8 dihydroxyflavone (17). These early reports may lead to future interventions to mitigate the effects of the CSA and CSB, and potentially shed light on strategies for preventing the degenerative effects of aging and oxidative stress related noise induced hearing loss.

\section{Bibliography}

1. Nance MA, Berry SA. Cockayne syndrome: review of 140 cases. Am J Med Genet. 1992; 42(1):6884. [PubMed: 1308368] 
2. Assfalg R, Alupei MC, Wagner M, Koch S, Gonzalez OG, Schelling A, Scharffetter-Kochanek K, Iben S. Cellular sensitivity to UV-irradiation is mediated by RNA polymerase I transcription. PLoS One. 2017; 12(6):e0179843. [PubMed: 28636660]

3. Hanawalt PC, Spivak G. Transcription-coupled DNA repair: two decades of progress and surprises. Nat Rev Mol Cell Biol. 2008; 9(12):958-70. [PubMed: 19023283]

4. Chatre L, Biard DS, Sarasin A, Ricchetti M. Reversal of mitochondrial defects with CSB-dependent serine protease inhibitors in patient cells of the progeroid Cockayne syndrome. Proc Natl Acad Sci U S A. 2015; 112(22):E2910-9. [PubMed: 26038566]

5. Vessoni AT, Herai RH, Karpiak JV, Leal AM, Trujillo CA, Quinet A, Agnez Lima LF, Menck CF, Muotri AR. Cockayne syndrome-derived neurons display reduced synapse density and altered neural network synchrony. Hum Mol Genet. 2016; 25(7):1271-80. [PubMed: 26755826]

6. Merchant, SN., Joseph, B., Nadol, JB, Jr. Schuknecht's Pathology of the Ear. 3. PMPH-USA: 2010. p. 29

7. Wilson BT, Stark Z, Sutton RE, Danda S, Ekbote AV, Elsayed SM, Gibson L, Goodship JA, Jackson AP, Keng WT, King MD, McCann E, Motojima T, Murray JE, Omata T, Pilz D, Pope K, Sugita K, White SM, Wilson IJ. The Cockayne Syndrome Natural History (CoSyNH) study: clinical findings in 102 individuals and recommendations for care. Genet Med. 2016; 18(5):483-93. [PubMed: 26204423]

8. Karikkineth AC, Scheibye-Knudsen M, Fivenson E, Croteau DL, Bohr VA. Cockayne syndrome: Clinical features, model systems and pathways. Ageing Res Rev. 2017; 33:3-17. [PubMed: 27507608]

9. Falik-Zaccai TC, Laskar M, Kfir N, Nasser W, Slor H, Khayat M. Cockayne syndrome type II in a Druze isolate in Northern Israel in association with an insertion mutation in ERCC6. Am J Med Genet A. 2008; 146A(11):1423-9. [PubMed: 18446857]

10. Laugel V. Cockayne syndrome: The expanding clinical and mutational spectrum. Mechanisms of Ageing and Development. 2013; 134(5):161-170. [PubMed: 23428416]

11. Shemen LJ, Mitchell DP, Farkashidy J. Cockayne syndrome-an audiologic and temporal bone analysis. Am J Otol. 1984; 5(4):300-7. [PubMed: 6720882]

12. Gandolfi A, Horoupian D, Rapin I, DeTeresa R, Hyams V. Deafness in Cockayne's syndrome: morphological, morphometric, and quantitative study of the auditory pathway. Ann Neurol. 1984; 15(2):135-43. [PubMed: 6703654]

13. Rapin I, Weidenheim K, Lindenbaum Y, Rosenbaum P, Merchant SN, Krishna S, Dickson DW. Cockayne syndrome in adults: review with clinical and pathologic study of a new case. J Child Neurol. 2006; 21(11):991-1006. [PubMed: 17092472]

14. Brooks PJ. Blinded by the UV light: how the focus on transcription-coupled NER has distracted from understanding the mechanisms of Cockayne syndrome neurologic disease. DNA Repair (Amst). 2013; 12(8):656-71. [PubMed: 23683874]

15. Nagtegaal AP, Rainey RN, van der Pluijm I, Brandt RM, van der Horst GT, Borst JG, Segil N. Cockayne syndrome group $\mathrm{B}(\mathrm{Csb})$ and group a (Csa) deficiencies predispose to hearing loss and cochlear hair cell degeneration in mice. J Neurosci. 2015 Mar 11; 35(10):4280-6. [PubMed: 25762674]

16. Rainey RN, Ng SY, Llamas J, van der Horst GT, Segil N. Mutations in Cockayne SyndromeAssociated Genes (Csa and Csb) Predispose to Cisplatin-Induced Hearing Loss in Mice. J Neurosci. 2016 Apr 27; 36(17):4758-70. [PubMed: 27122034]

17. Viana LM, Seyyedi M, Brewer CC, Zalewski C, DiGiovanna JJ, Tamura D, Totonchy M, Kraemer $\mathrm{KH}$, Nadol JB Jr. Histopathology of the inner ear in patients with xeroderma pigmentosum and neurologic degeneration. Otol Neuroto. 2013; 34(7):1230-6.

18. Wang Y, Jones-Tabah J, Chakravarty P, Stewart A, Muotri A, Laposa RR, Svejstrup JQ. Pharmacological Bypass of Cockayne Syndrome B Function in Neuronal differentiation. Cell Rep. 2016; 14(11):2554-61. [PubMed: 26972010] 


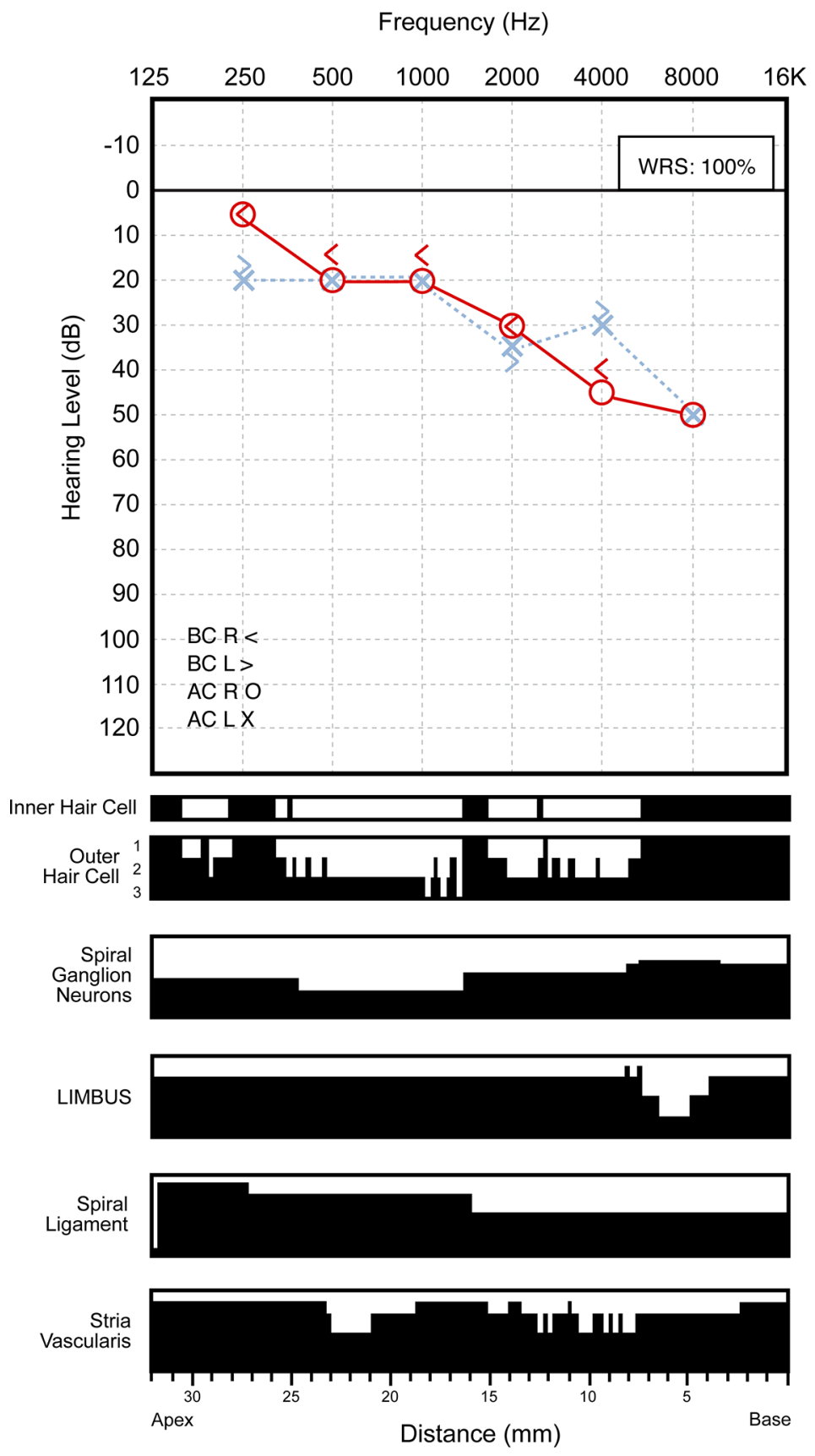

Figure 1.

Audiogram of both ears obtained five years prior to the donor's death, and a cytocochleogram of the right ear after death. Notable is the good hearing in the low frequencies with down-sloping SNHL and good word recognition scores. (WRS - word recognition scores, $\mathrm{BC}$ - bone conduction, $\mathrm{AC}$ - air conduction, $\mathrm{R}$ - right, $\mathrm{L}$ - Left). In addition to loss of hair cells and neurons, there was moderate to severe cellular loss in the limbus, spiral ligament, and stria vascularis. 


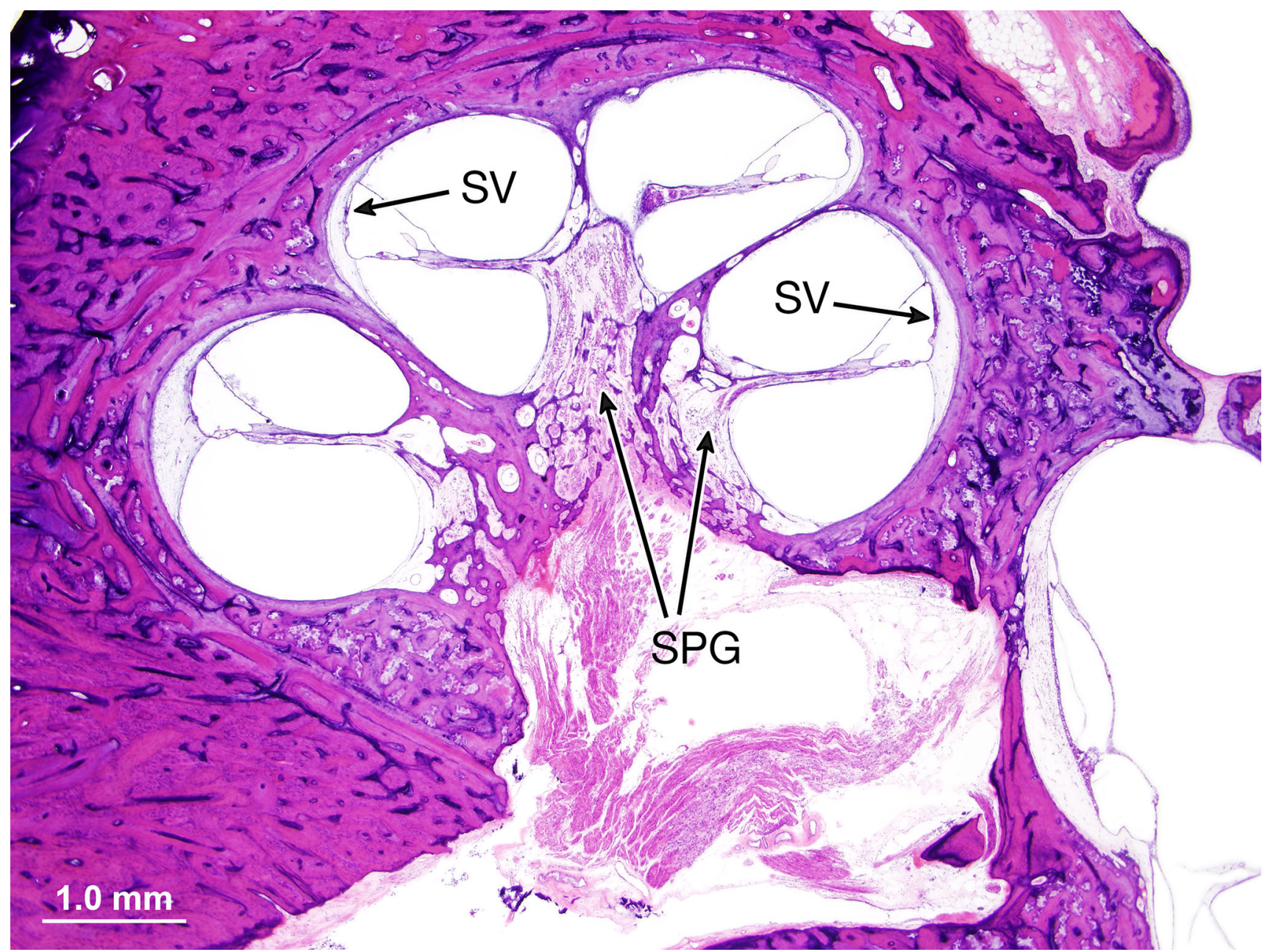

Figure 2.

Mid-modiolar section of the right cochlea. The density of spiral ganglion cells (SPG) is reduced in the basal turn. There is atrophy of the stria vascularis (SV) throughout the cochlea. 


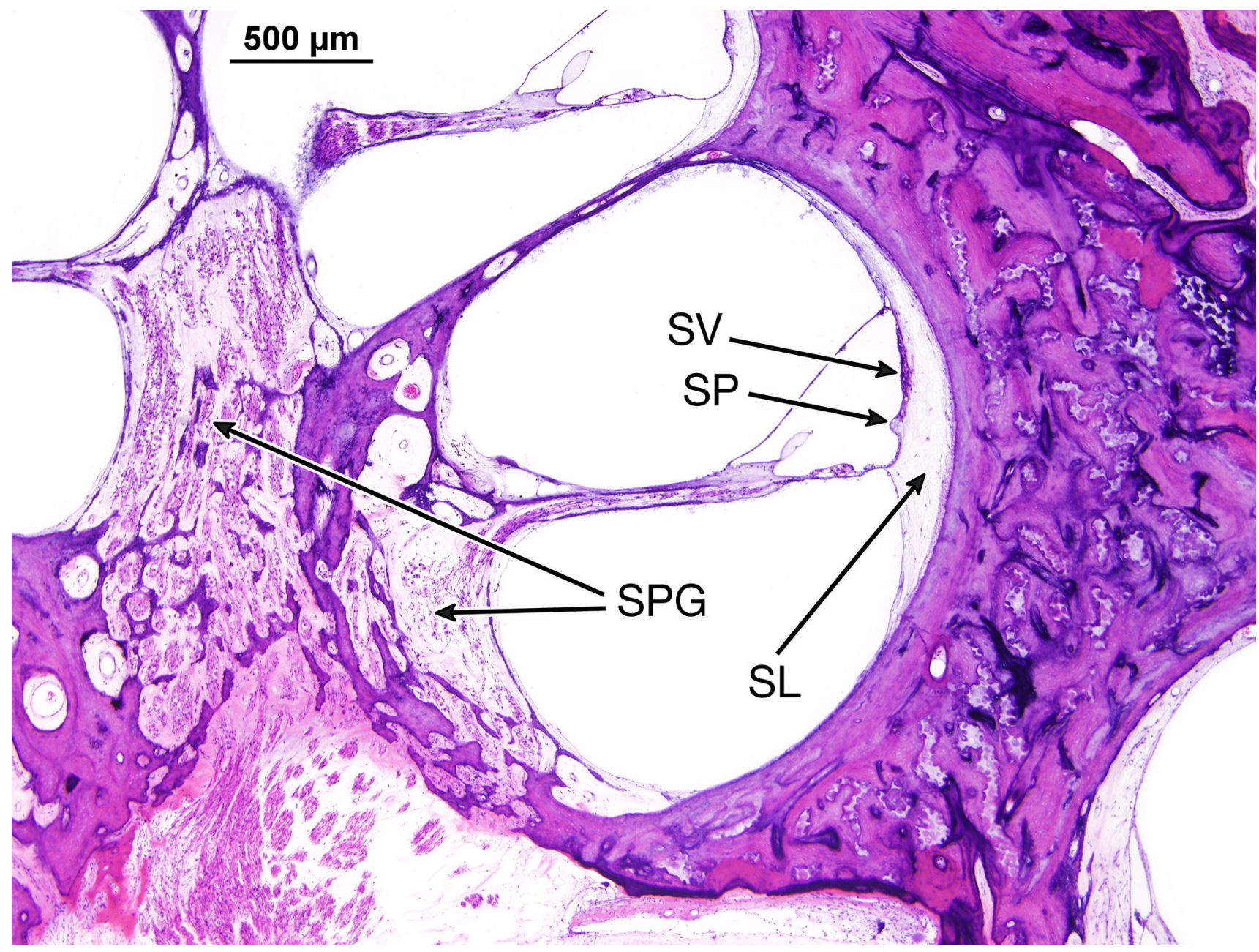

Figure 3.

The spiral ganglion cells (SPG) reduced in number in the basal turn. There is loss of cellularity of the spiral ligament (SL), stria vascularis (SV), and spiral prominence (SP). 


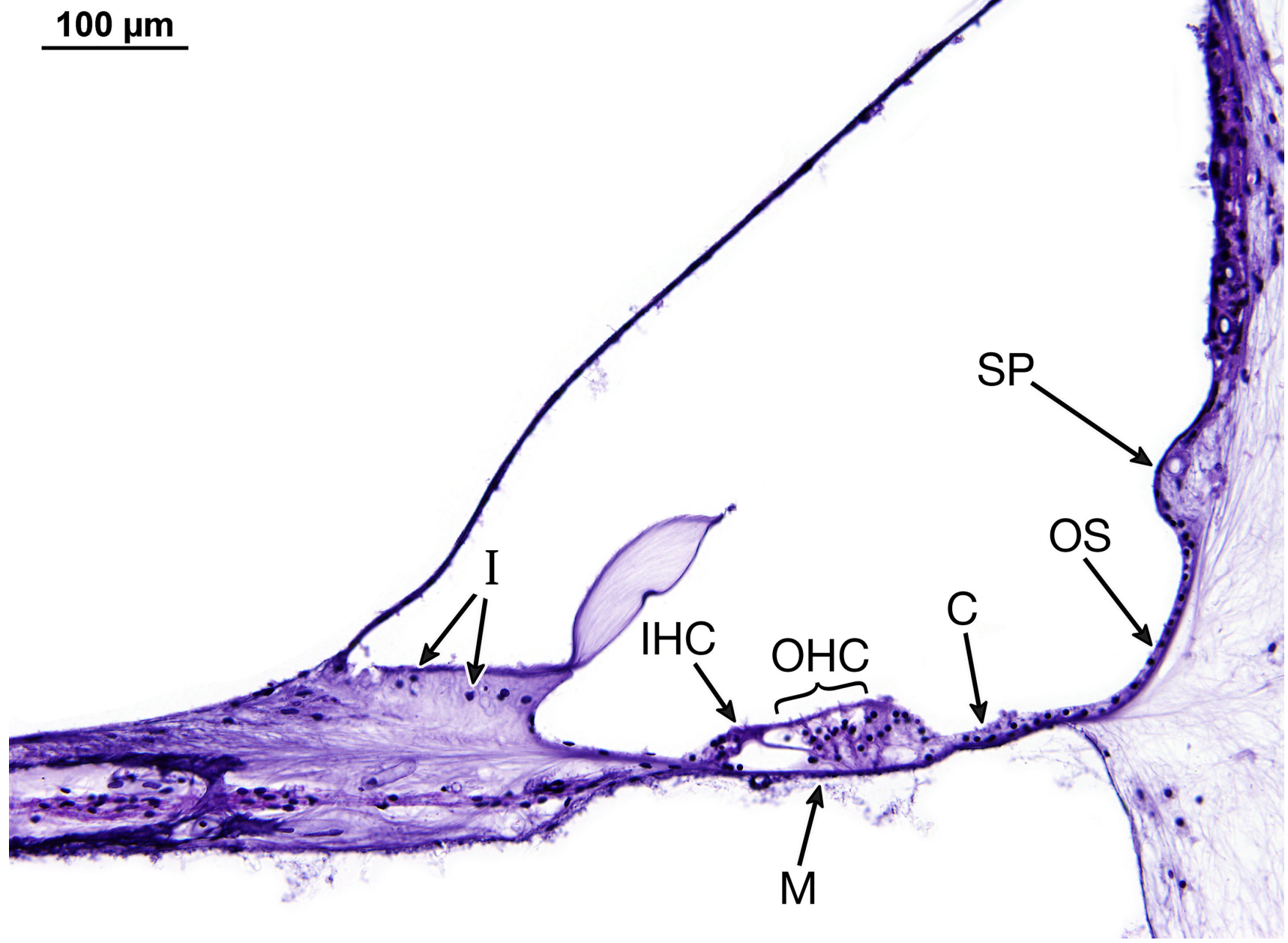

Figure 4.

The basal turn of the right cochlea. The inner (IHC) and outer (OHC) hair cells are preserved in this section. There is loss of cellularity of the interdental (I) cells, mesenchymal (M) cells below the basilar membrane, the Claudius (C) cells, outer sulcus (OS) cells, and spiral prominence (SP). 


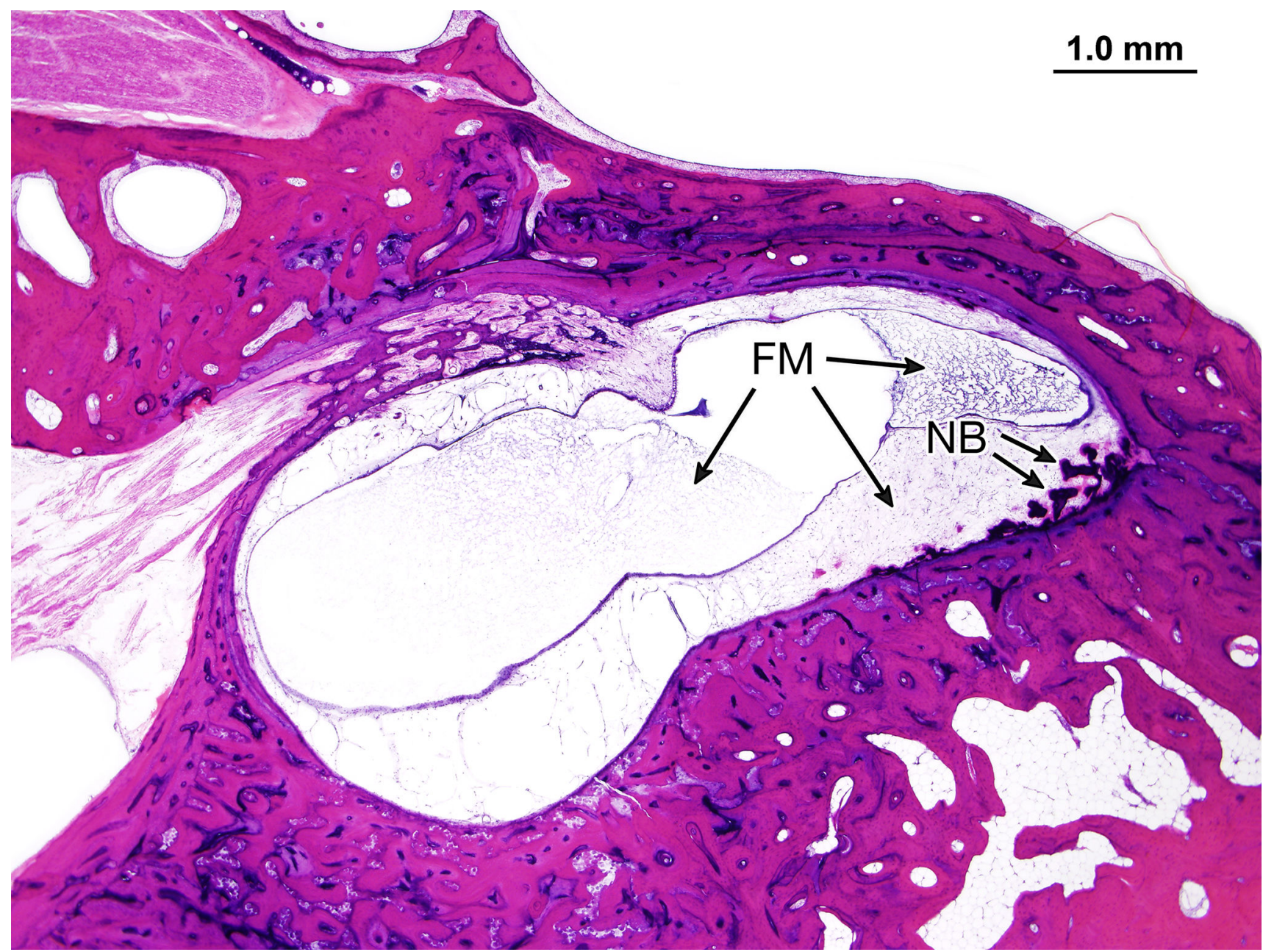

Figure 5.

Sectioned through the lateral semicircular canal of the right ear. There is fibrillar material (FM), both in the endolymphatic and perilymphatic spaces, and there is new bone (NB) formation in the perilymphatic space. (H \& E staining). 